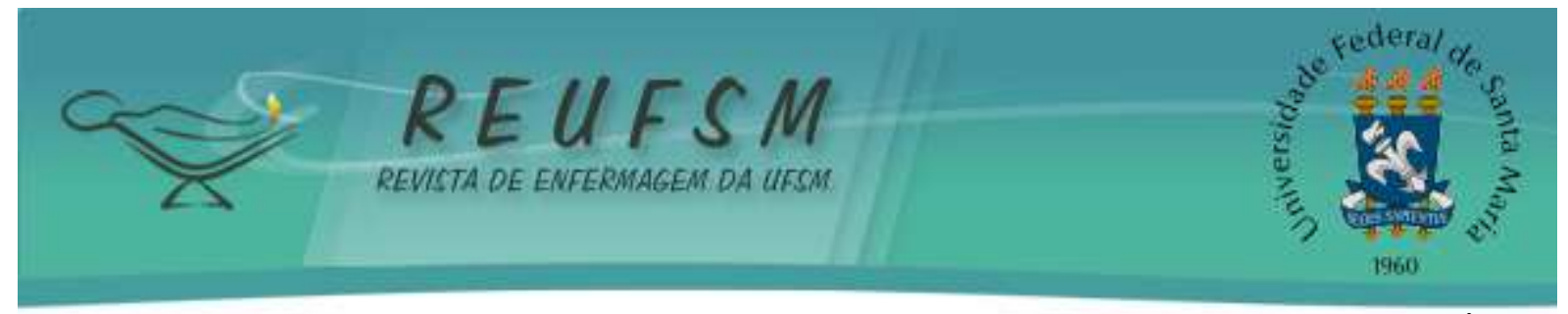

RELATO DE EXPERIÊNCIA

\title{
ATENÇÃO À SAÚDE DO TRABALHADOR: INSTRUMENTO PARA SUBSIDIAR A CONSULTA DE ENFERMAGEM
}

\section{ATTENTION TO THE WORKER'S HEALTH CARE: A TOOL TO SUBSIDIZE NURSING VISITS \\ ATENCIÓN A LA SALUD DEL TRABAJADOR: UNA HERRAMIENTA DE APOYO A LA CONSULTA DE ENFERMERÍA}

Doi: $10.5902 / 2179769219832$

\author{
Denise Cristina Alves de Moura ${ }^{1}$ \\ Paula Cristina Gomes ${ }^{2}$ \\ Gilvan Carlos Toledo Júnior ${ }^{3}$ \\ Nathália Alvarenga-Martins ${ }^{4}$ \\ Rosangela Maria Greco ${ }^{5}$
}

RESUMO: Objetivo: descrever a construção de impressos para coleta de dados, diagnósticos, prescrições e resultados de enfermagem para subsidiar a consulta a trabalhadores. Método: relato de experiência da construção de um instrumento para sistematizar a assistência de enfermagem na consulta ao trabalhador. Resultados: foram criados três impressos: o primeiro destinado ao histórico de enfermagem; segundo, lista de 26 diagnósticos de enfermagem e terceiro, lista de 22 intervenções de enfermagem e 23 resultados de enfermagem esperados, os dois últimos impressos alinhados segundo as taxonomias North American Nursing Diagnosis Assosciation International (NANDA I), Nursing Intervention Classification International (NIC), Nursing Outcome Classification (NOC). Considerações Finais: as contribuições para a enfermagem e a saúde do trabalhador foram: criação de um instrumento para subsidiar a consulta de enfermagem sistematizada a trabalhadores; atendimento à legislação vigente da implementação do processo de enfermagem.

Descritores: Enfermagem do trabalho; Processos de enfermagem; Saúde do trabalhador.

ABSTRACT: Aim: to describe the development of forms for data collection, diagnoses, prescriptions and nursing outcomes to support the nursing process during occupational health care. Method: account experience of building a tool to systematize nursing care in consultation with the worker. Results: three health data collection tools were created : the first one intended to register nursing track record; the second lists 26 nursing diagnoses and the third describes 22 possible nursing interventions and 23 Nursing expected results. The last two were aligned according to North American Nursing Diagnosis Assosciation International (NANDA I), Nursing Intervention Classification International (NIC), Nursing Outcome Classification (NOC) taxonomies. Conclusion: the contributions to nursing and to the worker health were: the creation of an instrument to subsidize the

\footnotetext{
1 Enfermeira. Doutoranda do Programa de Pós-Graduação em Saúde Coletiva. Mestre em Enfermagem. Especialista em Enfermagem do Trabalho. Faculdade de Enfermagem da Universidade Federal de Juiz de Fora (UFJF) Juiz de Fora, Minas Gerais. Brasil. Email: denisematipo@yahoo.com.br

${ }^{2}$ Enfermeira. Mestre em Enfermagem. Especialista em Enfermagem do Trabalho. Faculdade de Enfermagem da UFJF. Juiz de Fora, Minas Gerais. Brasil. Email: gomescrispaula@gmail.com

3 Enfermeiro. Especialista em Enfermagem do Trabalho. Juiz de Fora, Minas Gerais. Brasil. Email: gilvan_carlos@hotmail.com.br

${ }^{4}$ Enfermeira. Mestre em Enfermagem. Faculdade de Enfermagem da UFJF. Juiz de Fora, Minas Gerais. Brasil. Email: nath.alvarenga.martins@gmail.com

${ }^{5}$ Enfermeira. Doutora em Saúde Pública, Professora da Faculdade de Enfermagem da UFJF. Juiz de Fora, Minas Gerais. Brasil. Email: romagreco@gmail.com
} 


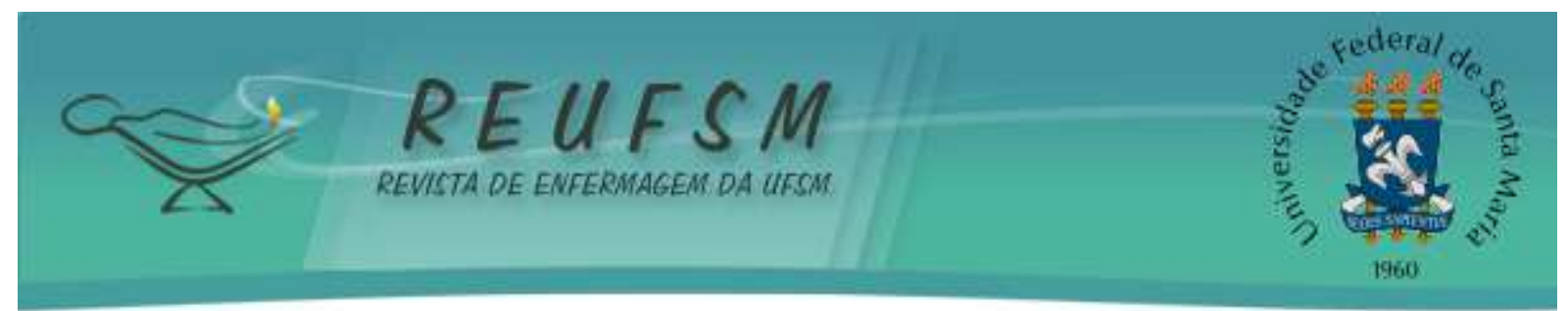

systematized nursing consultation to workers; compliance with current legislation on the implementation of the nursing process.

Descriptors: Occupational health nursing; Nursing process; Occupational health.

RESUMEN: Objetivo: describir la construcción de formularios para la recolección de datos, diagnósticos, recetas y resultados de enfermería para apoyar la consulta de los trabajadores. Método: relato de la experiencia de la construcción de una herramienta para sistematizar los cuidados de enfermería en consulta con el trabajador. Resultados: creado tres impresos: el primer, destinado a la historia de la enfermería; el segundo, lista de 26 diagnósticos de enfermería y el tercero, lista de 22 intervenciones de enfermería y 23 resultados de enfermería esperados, los dos ultimos impresos fueron ordenados por las taxonomías North American Nursing Diagnosis Asociación Internacional (NANDA I), la Clasificación Internacional de Intervención de Enfermería (NIC), Resultado de Enfermería (Clasificación NOC). Consideraciones Finales: contribuciones para la enfermería y para la salud del trabajador fueron: la creación de un instrumento de apoyo a la consulta de enfermeira sistematizada a los trabajadores; el cumplimiento de la legislación vigente a la aplicación del proceso de enfermería.

Descriptores: Enfermería del trabajo; Procesos de enfermería; Salud laboral.

\section{INTRODUÇÃO}

A Saúde do trabalhador é um campo da Saúde pública cujo foco reside no processo de trabalho e na defesa de mudanças nas condições potencialmente causadoras de adoecimento. Além disso, valoriza o saber e a experiência do trabalhador sobre seu próprio trabalho. ${ }^{1-3}$

O campo da saúde do trabalhador possui abordagem multidisciplinar e multiprofissional e dentre essas várias profissões que atuam e contribuem para este campo, destaca-se a enfermagem, cujo objeto de trabalho é o cuidado, com finalidade de promover a saúde integral do ser humano e comunidade, bem como prevenir agravos. Para tanto, faz-se necessária a instrumentalização de profissionais para a execução de ações voltadas à promoção e à proteção da saúde, com vistas a reduzir as morbimortalidades decorrentes das ações produtivas empregadas no trabalho..$^{4-9}$

O Conselho Federal de Enfermagem (COFEN), por meio da resolução 358/2009, determina o uso da Sistematização da Assistência de Enfermagem (SAE) e a implementação do Processo de Enfermagem (PE) nos ambientes em que o cuidado de enfermagem é realizado. Esta resolução orienta o registro, a comunicação e a documentação da assistência. ${ }^{10-14}$ Segundo a referida legislação, é preconizado que o PE seja organizado conforme um referencial teórico-filosófico capaz de direcionar o raciocínio clínico do enfermeiro, a partir da teoria escolhida. ${ }^{12-13}$

Diante das considerações acima apresentadas, surgiram as seguintes questões norteadoras levantadas pelos pesquisadores: Como conciliar o objeto de enfermagem pertinente à saúde do trabalhador com as taxonomias de diagnósticos, intervenções e resultados de enfermagem? Como basear a prática de enfermagem do trabalho de acordo com a legislação vigente que aborda a SAE a partir de uma teoria de enfermagem?

Assim, o presente estudo tem como objetivo descrever a construção de impressos para coleta de dados, diagnósticos, prescrições e resultados de enfermagem para subsidiar a consulta a trabalhadores.

Este estudo se justifica por possibilitar: o registro/documentação da assistência de enfermagem na consulta ao trabalhador; padronizar a linguagem nos registros de enfermagem por meio das taxonomias American Nursing Diagnosis Assosciation 


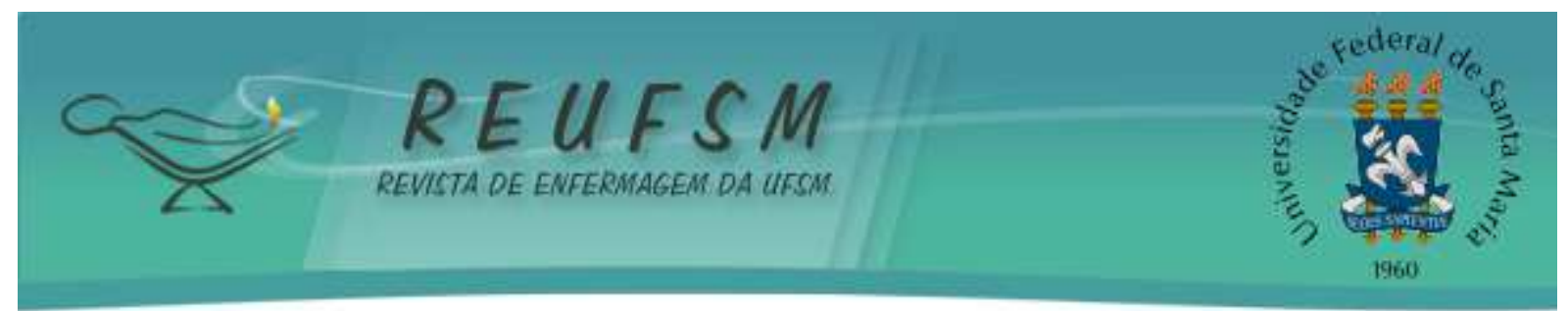

International (NANDA I), Nursing Intervention Classification (NIC) e Nursing Outcome Classisfication (NOC), conhecidas como NANDA, NIC e NOC (NNN); ${ }^{14-16}$ respaldar legalmente as ações da enfermagem, estando em consonância com a Resolução 358/2009 do COFEN; ${ }^{12}$ além de possibilitar visibilidade e reconhecimento para esta profissão.

\section{MÉTODOS}

Trata-se de um relato de experiência da descrição da construção de um instrumento para sistematizar a consulta de enfermagem a trabalhadores. 0 instrumento construído é constituído por três impressos que subsidiam os registros do histórico de enfermagem e consolidam os diagnósticos, intervenções e resultados segundo as taxonomias NNN.

O estudo foi desenvolvido por quatro enfermeiros pesquisadores atuantes na área de saúde do trabalhador e por uma enfermeira pesquisadora com experiência prévia na criação de modelos para sistematizar a assistência de enfermagem segundo as taxonomias NNN. O instrumento foi criado durante a realização de pós-graduação Lato sensu em Enfermagem do Trabalho, nos anos 2014 e 2015.

Para o levantamento dos principais diagnósticos de enfermagem, utilizou-se a experiência clínica dos autores e o levantamento bibliográfico dos elementos mais comuns encontrados no processo saúde-doença dos trabalhadores em situação de consulta de enfermagem. Elementos estes que fossem capazes de traduzir as situações reais ou potenciais de saúde (diagnósticos) e a partir daí viriam a nortear os cuidados de enfermagem necessários (intervenções), bem como as formas de avaliação desses cuidados (avaliação dos resultados esperados). A metodologia é descrita pela literatura como método indutivo/dedutivo e intuitivo. ${ }^{17-18}$

Para a padronização da linguagem e elaboração do instrumento proposto foram utilizadas as taxonomias NANDA I, NIC e NOC, para elaboração dos diagnósticos, intervenções e resultados de enfermagem, respectivamente. ${ }^{14-16}$

A necessidade de ofertar uma abordagem de enfermagem capaz de atender às necessidades fisiológicas, psicológicas, socioculturais, desenvolvimentais e espirituais do trabalhador, foi determinante para a escolha da Teoria de Betty Neuman ${ }^{19-20}$, para organizar e orientar o processo de enfermagem. Assim, o instrumento foi construído em consonância com a legislação em vigor. ${ }^{12}$

\section{RESULTADOS E DISCUSSÃO}

0 instrumento desenvolvido para operacionalizar a sistematização da consulta de enfermagem no contexto da saúde do trabalhador se constitui de três impressos, sendo o primeiro destinado à coleta de dados; o segundo aos possíveis diagnósticos de enfermagem e o último um consolidado das intervenções e dos resultados de enfermagem esperados.

0 impresso de coleta de dados (Figura 1) foi elaborado para subsidiar a realização do histórico de enfermagem.

\begin{tabular}{|l|l|}
\hline \multicolumn{2}{|l|}{ INSTRUMENTO DE COLETA DE DADOS SOBRE O TRABALHADOR E SUAS CONDIÇÕES LABORAIS } \\
\hline Nome: & Sexo: \\
\hline Data Nascimento: & Renda: \\
\hline Estado civil: & Escolaridade (anos): \\
\hline Número de dependentes familiar: & Chefe de família: \\
\hline Cor pele: & Espiritualidade: \\
\hline Número de filhos:
\end{tabular}



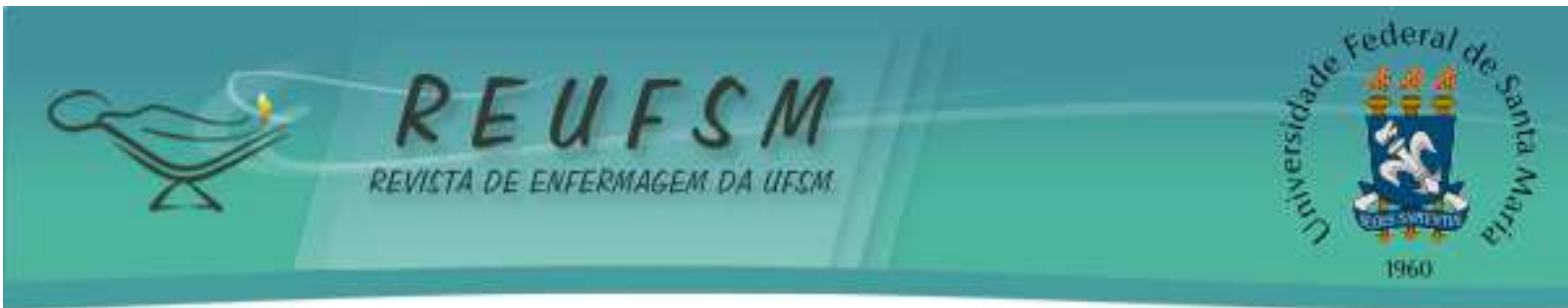

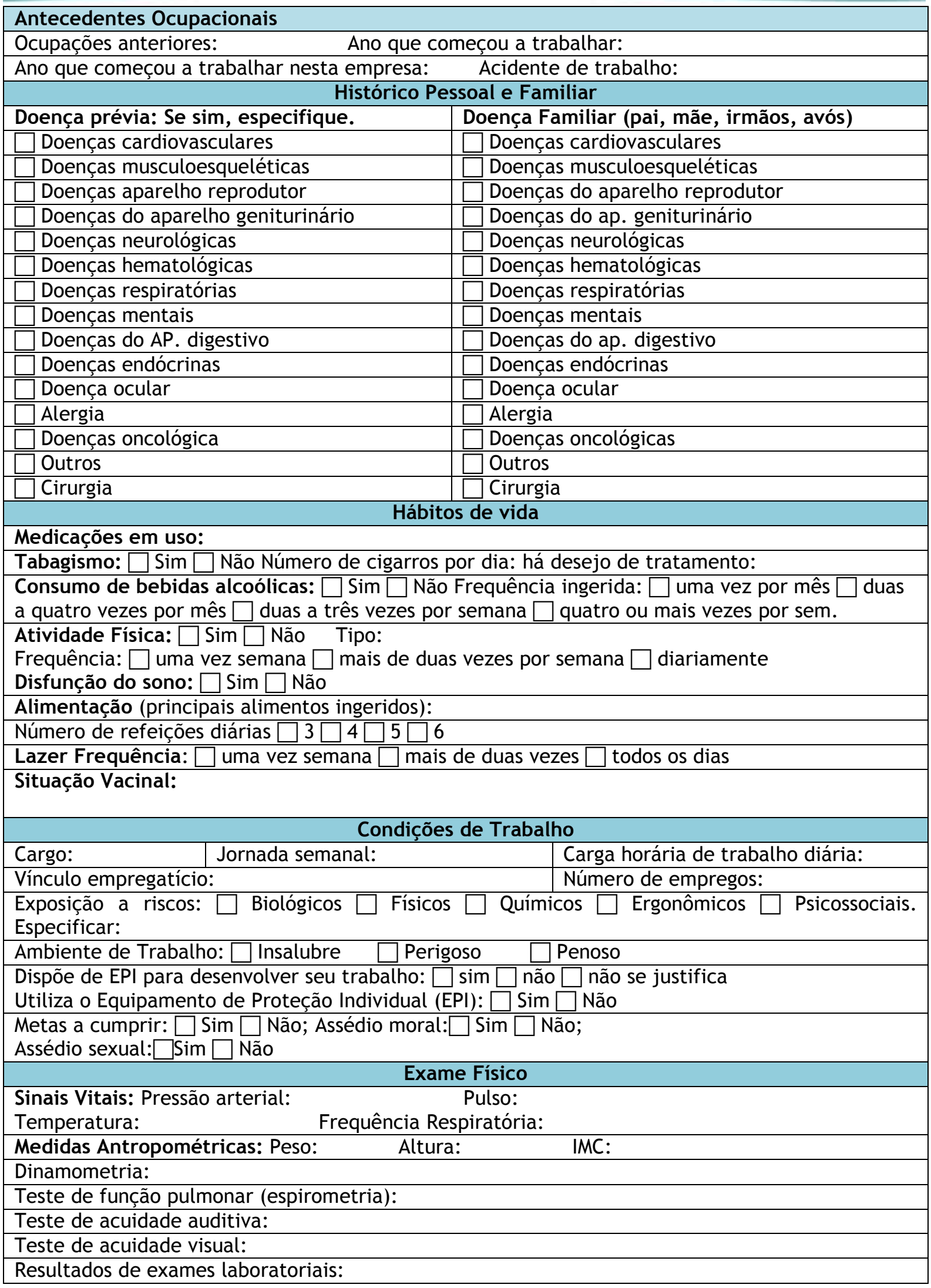




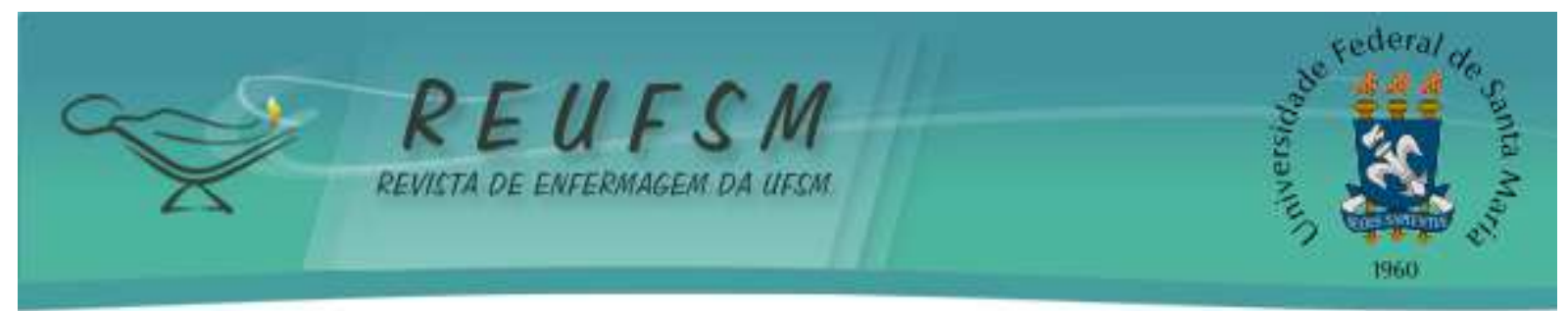

Figura 1: Impresso para subsidiar a coleta de dados do histórico da primeira consulta de enfermagem ao trabalhador, Juiz de Fora, outubro. 2015.

Segundo a Teoria de Betty Neuman, o indivíduo está sujeito a estressores que podem ser: de origem intrapessoal (originados do próprio comportamento do individuo); de origem interpessoal (que se manifestam na interação com o outro) e transpessoais (representadas pelas condições do ambiente). ${ }^{19}$ Estes estressores podem significar impactos no processo saúde-doença do trabalhador.

0 instrumento subsidia a realização do histórico de enfermagem, permitindo a identificação dos possíveis estressores que agem sobre os trabalhadores que estão em situação de risco. ${ }^{19-21}$ Também, o levantamento das necessidades individuais e coletivas, os problemas vivenciados e as reações humanas, direcionam as ações de enfermagem e fornecem subsídios para o desenvolvimento das outras etapas do processo de enfermagem. ${ }^{21-23}$

Em consonância com o instrumento de coleta de dados apresentado, um estudo que analisa as condições de trabalho e vida a partir da teoria de Betty Neuman aponta como estressores e fatores protetores os quais o trabalhador está sujeito a sofrer interferências: estressores intrapessoais- idade, número de empregos, (ins)estabilidade, tempo de serviço; Interpessoais- situação conjugal, número de filhos, ser chefe de família; transpessoaiscondições de trabalho, gênero e grau de escolaridade. ${ }^{20}$

0 impresso que contempla os diagnósticos de enfermagem (Figura 2) apresenta 26 diagnósticos, segundo a NANDA I, selecionados como os diagnósticos nucleares mais comuns para abordagem de enfermagem no contexto de saúde do trabalhador. Cada diagnóstico aparece identificado com sua página, título, fatores relacionados/de risco e/ou suas características definidoras.

\section{LISTA DE DIAGNÓSTICO DE ENFERMAGEM AO TRABALHADOR}

LEGENDA: I= Diagnóstico Identificado A=Diagnóstico Acompanhado R= Diagnóstico Resolvido

\section{Nome:}

Data:

Estilo de vida sedentário (208p.): relacionado a: $\square$ conhecimento deficiente sobre os benefícios que a atividade física traz à saúde $\square$ falta de interesse $\square$ falta de motivação $\square$

1 falta de recursos caracterizado por: $\square$ escolhe uma rotina diária sem atividade físicas demonstra falta de condicionamento físico $\square$ verbaliza preferência por atividades com poucos exercícios físicos

Disposição para estado de imunização melhorado (218p.) caracterizado por Expressa

2 desejo de: $\square$ reforçar a condição de imunização $\square$ reforçar o comportamento para prevenir doenças infecciosas $\square$ de reforçar o registro das imunizações

Nutrição desequilibrada: mais do que as necessidades corporais (231p.) relacionado a: ingestão excessiva em relação a atividade física $\square$ ingestão excessiva em relação as necessidades metabólicas caracterizado por: $\square$ comer em resposta a estímulos externos

3 comer em resposta a estímulos internos que não a fome(ansiedade) $\square$ dobra da pele do tríceps > que $25 \mathrm{~mm}$ em mulheres e $15 \mathrm{~mm}$ em homens $\square$ ingestão de alimentos concentrada ao final do dia $\square$ estilo de vida sedentário $\square$ padrão de alimentação disfuncional $\square$ peso $20 \%$ acima da altura e compleição

Nutrição desequilibrada: menos que as necessidades corporais (233p.) relacionado a $\square$ fatores psicológicos caracterizado por $\square$ aversão ao ato de comer $\square$ falta de interesse na

4 comida $\square$ ideias erradas $\square$ perda de peso com ingestão adequada da comida $\square$ peso corporal $20 \%$ ou mais abaixo do ideal $\square$ relato de ingestão inadequada de alimentos, menor que a porção diária recomendada

5 Risco de glicemia instável (237p.) fatores de risco $\square$ conhecimento deficiente sobre o




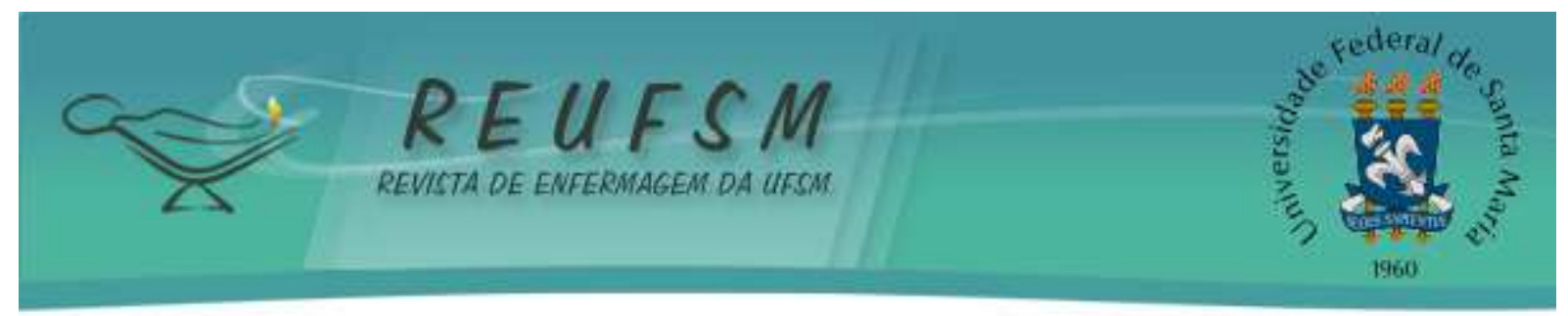

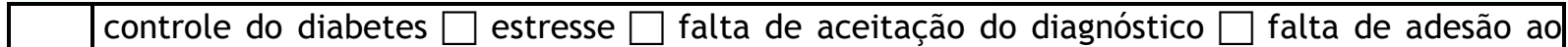
controle do diabetes

Insônia (273p.) relacionado a: $\square$ ansiedade $\square$ depressão $\square$ estresse $\square$ fatores ambientais $\square$ ingestão de álcool $\square$ fatores ambientais (ruído, luz, temperatura ambiental, umidade, ambiente desconhecido $\square$ higiene do sono inadequado $\square$ medicamentos $\square$ ingestão de estimulantes $\square$ medo $\square$ prejuízo do padrão de sono normal (viagens e turno de - trabalho)definido por: $\square$ aumento do absenteísmo $\square$ falta de energia observada $\square$ relato de acordar mais cedo $\square$ relato de aumento de acidentes $\square$ relato de dificuldade de concentração $\square$ relato de dificuldade para adoecer $\square$ relato de dificuldade para permanecer dormindo $\square$ relato de distúrbios do sono que provocam consequências no dia seguinte $\square$ relato de falta de energia $\square$ relato de insatisfação com sono $\square$ relato de mudança de humor $\square$ relato de qualidade de vida diminuída $\square$ relato de sono não restaurador

Privação de sono (276p.) relacionado a: $\square$ apneia do sono $\square$ assincronia circadiana sustentada $\square$ estimulação ambiental por tempo prolongado $\square$ higiene do sono inadequada 7 por tempo prolongado caracterizado por: $\square$ agitação $\square$ ansiedade $\square$ apatia $\square$ capacidade funcional diminuída $\square$ fadiga $\square$ incapacidade de concentrar-se $\square$ indiferença $\square$ inquietação $\square$ irritabilidade $\square$ letargia $\square$ mal estar $\square$ reação lenta $\square$ sensibilidade aumentada a dor $\square$ sonolência durante o dia

Fadiga (287p.) relacionado a: $\square$ barulho $\square$ luzes $\square$ temperatura $\square$ umidade $\square$ anemia $\square$ condição física debilitada $\square$ desnutrição $\square$ esforço físico aumentado $\square$ gravidez $\square$ privação de sono $\square$ ansiedade $\square$ depressão $\square$ estresse $\square$ estilo de vida enfadonho $\square$ eventos negativos na vida $\square$ ocupação caracterizado por: $\square$ aumento das queixas físicas $\square$ concentração comprometida $\square$ desatento $\square$ desempenho diminuído $\square$ desinteresse quanto ao ambiente que o cerca $\square$ falta de energia $\square$ introspecção $\square$ letárgico $\square$ necessidade de energia adicional para realizar tarefas de rotina $\square$ cansaço $\square$ relato de cansaço $\square$ relato de incapacidade de manter as rotinas pessoais $\square$ relato de sentimentos de culpa por não cumprir com as suas responsabilidades $\square$ relato de incapacidade de restaurar energia mesmo após o sono $\square$ relato de uma constante falta de energia $\square$ sonolenta

Síndrome do estresse por mudança (399p.) relacionado a: $\square$ estado de saúde diminuído $\square$ falta de um sistema adequado de apoio $\square$ isolamento $\square$ mudança de um ambiente para outro

$9 \square$ relato de sentimento de impotência $\square$ perdas caracterizado por: $\square$ alienação $\square$ ansiedade $\square$ aumento de doença $\square$ baixa autoestima crônica $\square$ baixa autoestima situacional $\square$ dependência $\square$ depressão $\square$ distúrbios do padrão do sono $\square$ frustração $\square$ insegurança $\square$ isolamento $\square$ medo $\square$ perda de identidade $\square$ preocupação $\square$ solidão

Risco de síndrome do estresse por mudança (400p.) fatores de risco: $\square$ estado de saúde

10 diminuído $\square$ falta de um sistema adequado de apoio $\square$ mudança de um ambiente para outro $\square$ relato de sentimento de impotência $\square$ perdas

Ansiedade (404p.) relacionado a: $\square$ abuso de substâncias $\square$ ameaça ao estado de saúde estresse $\square$ exposição a toxinas $\square$ mudança no estado de saúde $\square$ mudança no ambiente necessidades não satisfeitas caracterizado por: afetivas $\square$ afobado $\square$ apreensivo angustiado $\square$ desesperado $\square$ incerteza $\square$ irritabilidade $\square$ medo $\square$ nervoso $\square$ preocupado $\square$

11 atenção prejudicada $\square$ bloqueio de pensamento $\square$ capacidade diminuída de solucionar problemas $\square$ confusão $\square$ dificuldade para concentrar-se $\square$ ruminação comportamentais $\square$ inquietação $\square$ insônia $\square$ nervosismo $\square$ relato de preocupações devido a mudança em eventos da vida $\square$ produtividade diminuída fisiológicas $\square$ aumento da tensão $\square$ diarréia $\square$ distúrbio do sono $\square$ dor abdominal $\square$ fadiga $\square$ náuseas $\square$ palpitação $\square$ pulso aumentado $\square$ respiração aumentada

Enfrentamento ineficaz (418p.) relacionado a: $\square$ crise $\square$ incerteza $\square$ suporte social inadequado criado pelas características dos relacionamentos caracterizado por: $\square$ abuso de agentes químicos $\square$ alta taxa de doença $\square$ comportamento destrutivo em relação a si mesmo

$12 \square$ comportamento destrutivo em relação aos outros $\square$ concentração insatisfatória $\square$ dificuldade para organizar informações $\square$ distúrbios no padrão de sono $\square$ fadiga $\square$ falta de comportamento direcionado a resolução de problemas $\square$ incapacidade de lidar conforme as informações $\square$ incapacidade de satisfazer as necessidades básicas 


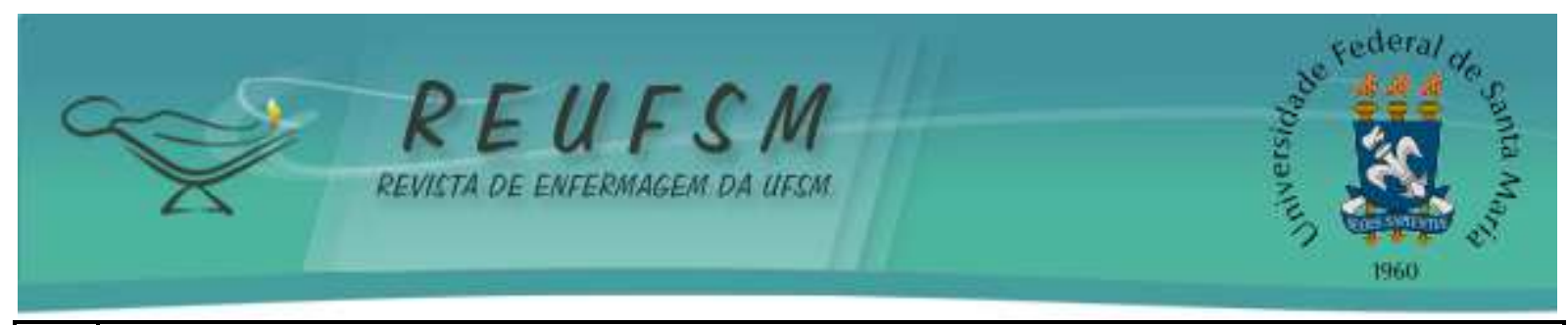

Sobrecarga de estresse (420p) relacionado a: $\square$ Múltiplos estressores concomitantes (ex. ameaças/exigências físicas, ameaças/exigências sociais) $\square$ recursos inadequados (ex., financeiros, sociais, educacionais/nível de conhecimento) caracterizado por: $\square$ relata

13 aumento dos sentimentos de impaciência $\square$ relata estresse situacional excessivo $\square$ relata impacto negativo em decorrência do estresse (ex. sintomas físicos, sofrimento psicossocial, sensação de "estar doente" ou de "estar para adoecer") $\square$ relata problemas com tomada de decisões $\square$ relata sensação de tensão

Sofrimento Moral (478p) relacionado a: $\square$ conflito entre tomadores de decisão $\square$ distância física do tomador de decisão $\square$ informações conflitantes que orientam tomada de decisões éticas $\square$ informações conflitantes que orientam tomada de decisões morais $\square$ perda de autonomia

Risco de infecção (485p.) fatores de risco: $\square$ aumento da exposição ambiental a patógenos $\square$ conhecimento deficiente para evitar exposição a patógenos $\square$ vacinação inadequada

Integridade da pele prejudicada (494p.) relacionado a: externos $\square$ fatores mecânicos (p.ex., forças abrasivas, pressão, contenção) $\square$ hipertermia $\square$ hipotermia $\square$ radiação substância química $\square$ umidade internos $\square$ circulação prejudicada caracterizado por: destruição de camadas da pele $\square$ rompimento de superfície do corpo

Risco de integridade da pele prejudicada (495p.) fatores de risco: externos $\square$ fatores mecânicos (p.ex., forças abrasivas, pressão, contenção) $\square$ hipertermia $\square$ hipotermia radiação $\square$ substância química $\square$ umidade internos $\square$ circulação prejudicada

Integridade tissular prejudicada (496p.) relacionado a: $\square$ extremos de temperatura $\square$

18 fatores mecânicos (p.ex., pressão, abrasão, fricção) $\square$ irritantes químicos $\square$ radiação caracterizado por: $\square$ tecido destruído $\square$ tecido lesado

Risco de lesão (497p.) fatores de risco: externos $\square$ biológicos (p.ex., nível de imunização da comunidade, microorganismos) $\square$ físicos (p. ex., projeto, estrutura e organização da comunidade, do edifício e/ou equipamento) $\square$ químicos (p. ex., poluentes, venenos, drogas, agentes farmacêuticos, álcool, nicotina, preservativos, cosméticos, corantes) internos perfil sanguíneo anormal

Risco de quedas (505p.) fatores de risco: ambientais $\square$ ambiente com móveis e objetos em excesso $\square$ condições climáticas (p. ex., pisos molhados, gelo) $\square$ pouca iluminação $\square$ tapetes espalhados pelo chão fisiológicos $\square$ dificuldades auditivas $\square$ dificuldades visuais neoplasias (p. ex., fadiga/mobilidade limitada) $\square$ problemas nos pés medicamentos agentes ansiolíticos $\square$ agentes anti-hipertensivos $\square$ antidepressivos tricíclicos $\square$ diuréticos hipnóticos $\square$ tranquilizantes $\square$ uso de álcool

Risco de trauma (513p.) fatores de risco: externos $\square$ aparelhos elétricos com defeito $\square$ combustíveis armazenados inadequadamente $\square$ contato com frio intenso $\square$ contato com máquinas de movimento rápido $\square$ contato com substâncias corrosivas $\square$ dirigir veiculo mecanicamente inseguro $\square$ exposição a máquinas perigosas $\square$ exposição excessiva a radiação $\square$ falta de proteção de fontes de calor $\square$ fios elétricos desgastados $\square$ pisos escorregadios $\square$ realizar experiências com produtos químicos $\square$ utilização inadequada de proteção necessária para a cabeça $\square$ vazamento de gás potencialmente inflamável internos $\square$ conhecimento deficiente em relação a precauções de segurança $\square$ conhecimento deficiente em relação a procedimentos de segurança $\square$ dificuldades cognitivas $\square$ história de trauma prévio $\square$ visão insatisfatória

Contaminação (523p.) relacionado a: externos $\square$ contaminação química da água $\square$ contato sem proteção com agentes químicos $\square$ contato sem proteção com metais pesados $\square$ exposição a radiação $\square$ exposição por ingestão de material radioativo $\square$ falta de roupas de proteção $\square$ serviços municipais inadequados (remoção do lixo, instalações para tratamento do 22 esgoto) $\square$ uso de contaminantes ambientais em casa (p. ex., pesticidas, agentes químicos, fumaça de cigarro) $\square$ uso inadequado de roupas de proteção internos $\square$ doenças preexistentes $\square$ exposições anteriores $\square$ exposições concomitantes caracterizado por: pesticidas $\square$ efeitos dermatológicos/gastrintestinais/neurológicos/pulmonares/renais de exposição a pesticidas produtos químicos $\square$ efeitos dermatológicos/gastrintestinais/imunológicos/neurológicos/pulmonares/renais de exposição a 


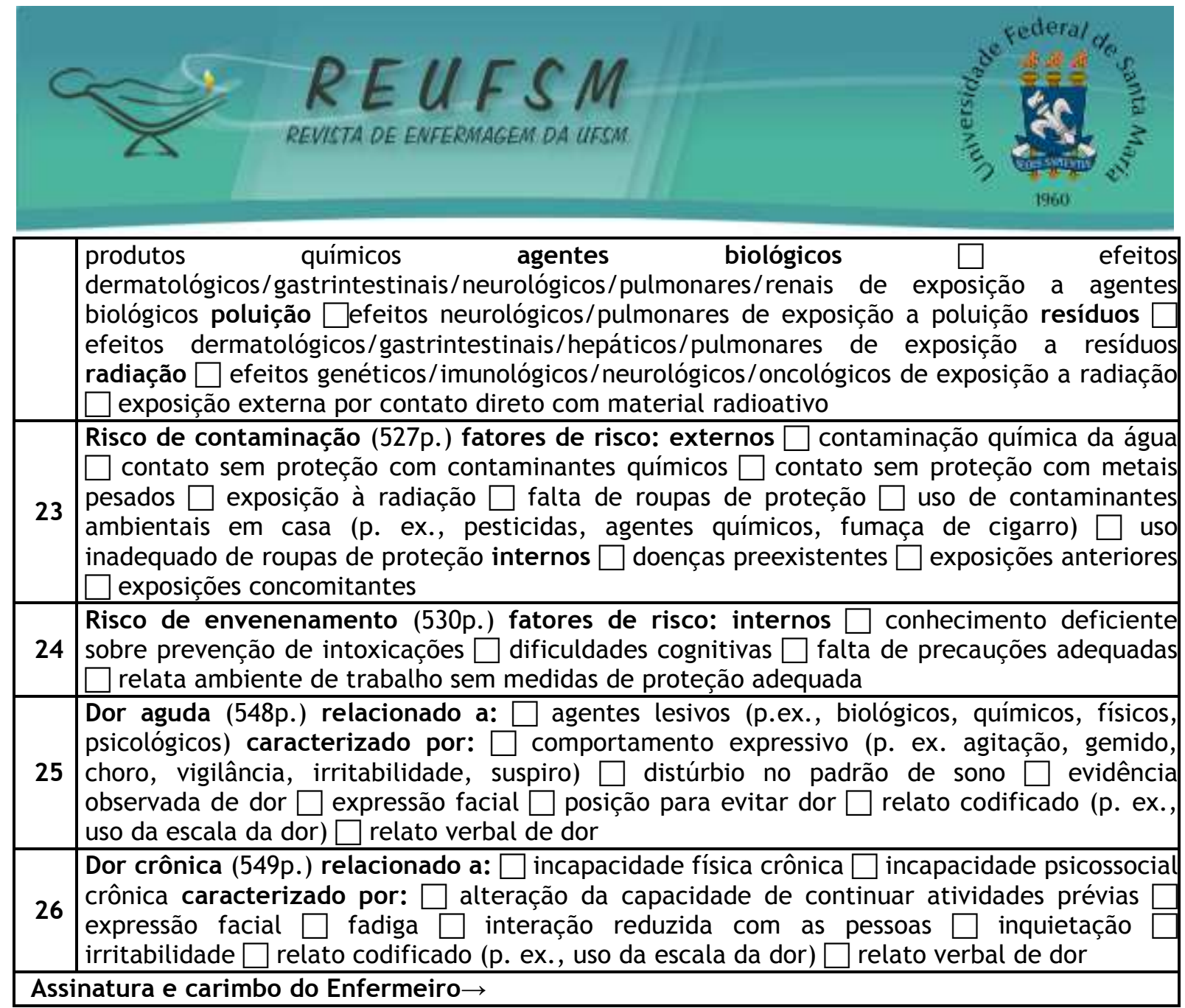

Figura 2 Lista de diagnóstico de enfermagem ao trabalhador. Juiz de Fora, outubro, 2015.

Os diagnósticos de enfermagem, segunda fase do processo, são julgamentos clínicos acerca das repostas dos indivíduos, família e comunidade aos estressores (internos ou externos). Estes podem gerar problemas reais e/ou potenciais de saúde. ${ }^{14,19,22}$

A etapa do planejamento de enfermagem se inicia logo após o levantamento dos diagnósticos e é concluída com o registro do plano de cuidados. Ela envolve estabelecimento de prioridades e resultados, determinação de prescrições de enfermagem, registro e documentação do plano de cuidados. ${ }^{12,21}$

0 terceiro impresso (figura 3 ) diz respeito às intervenções de enfermagem, segundo NIC, e estão alocadas junto às avaliações dos resultados esperados, segundo NOC. As intervenções totalizam 22, já os resultados estão em total de 23 e estão precedidos por uma escala tipo likert de cinco pontos.

\begin{tabular}{|c|c|}
\hline \multicolumn{2}{|c|}{$\begin{array}{c}\text { Instrumento de Intervenções e Avaliações dos Resultados de Enfermagem segundo NIC e NOC } \\
\text { para abordagem ao trabalhador }\end{array}$} \\
\hline Intervenções de Enfermagem segundo NIC & \begin{tabular}{|l|} 
Resultados de Enfermagem segundo NOC* \\
\end{tabular} \\
\hline$\square$ Controle de imunização/vacinação (p.463) & Comportamento de segurança \\
\hline Controle do peso (p.593) & pessoal (p.255) \\
\hline Melhora do enfrentamento (p.334) & Comportamento de imunização (p.241) \\
\hline Controle da hiperglicemia (p. 441) & Comportamento de ganho de peso (p239) \\
\hline Controle da hipoglicemia (p. 448) & Comportamento de manutenção de \\
\hline Melhora do sono (p.696) & peso $(p .243)$ \\
\hline Redução da ansiedade (p.156) & Comportamento de perda peso (p.245) \\
\hline Apoio emocional (p.328) & Enfrentamento (p.445) \\
\hline Assistência para parar de fumar (p. 414) & Conhecimento: controle do diabetes (p.295) \\
\hline Treinamento da memória (p.528) & Sono (p.712) \\
\hline Melhora da socialização (p.690) & Autocontrole da ansiedade (p.171) \\
\hline
\end{tabular}




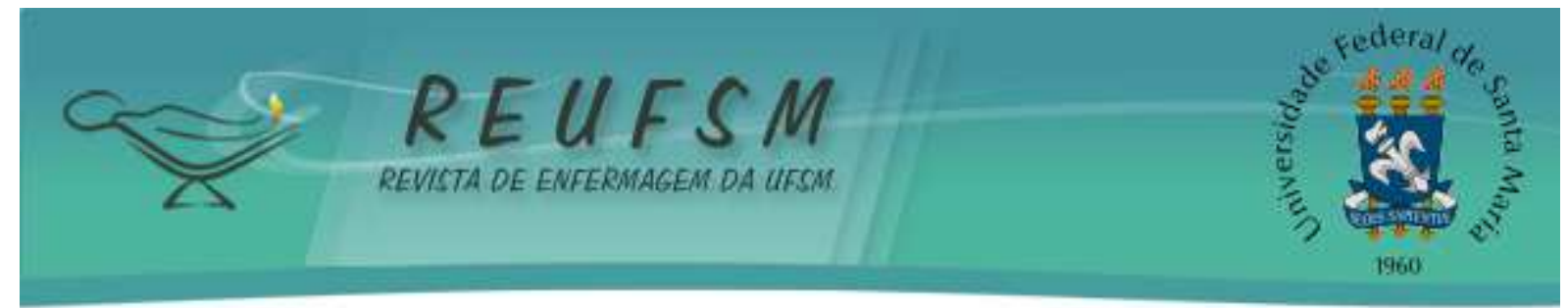

$\square$ Modificação do comportamento: habilidades sociais (p.236)

$\square$ Melhora da imagem corporal (p.457)

Mediação de conflitos (p.241)

Apoio à proteção contra abuso (p.95) Controle do ambiente: segurança do trabalhador (p.143)

$\square$ Identificação de risco (p. 653)

Gerenciamento de protocolo de emergência (p.326)

Controle de infecção (p.468)

Controle da alergia (p.122)

Cuidados com lesões: queimaduras (p.490)

Prevenção do suicídio (p.699)
Conhecimento: controle do uso de substâncias (p.298)

Controle de risco: uso de drogas (p.381)

Cognição (p.221)

Comportamento de cessação de fumar (p.235)

Memória (p.564)

Processamento de informações (p.632)

Habilidades de interação social (p.549)

Identidade (p.553)

Proteção contra abuso (p.633)

Recuperação de abuso (p.638)

Controle de riscos (p.361)

Detecção do risco (p.433)

Conhecimento: Controle de infecção (p.291)

Vontade de viver (p.723)

*Avaliados segundo escala NOC

Figura 3: Instrumento contendo as intervenções e avaliações dos resultados de enfermagem ao trabalhador, Juiz de Fora, outubro, 2015.

A intervenção ou prescrição de enfermagem é a quarta etapa do processo, compreendendo ações para alcançar os resultados almejados. Na saúde ocupacional as intervenções podem ser pensadas individualmente e/ou coletivamente. Para tanto, o processo de enfermagem deve iniciar com a visita do enfermeiro aos locais de trabalho, para identificar os riscos ocupacionais a que os trabalhadores estão expostos. ${ }^{10,12,23}$

A última fase do processo de enfermagem consiste na avaliação dos resultados, que abrange as respostas dos trabalhadores e as ações de enfermagem. Esta avaliação contribui para a qualidade e continuidade da assistência e fornece informações da efetividade do plano de cuidados, auxiliando a replanejar a assistência. ${ }^{16,21}$

Segundo a Teoria de Betty Neuman o enfermeiro, a partir da aplicação do PE, deve ser capaz de manter a estabilidade do continum saúde-doença do trabalhador, com vistas a reduzir o impacto que as condições estressoras podem causar ao indivíduo em situacão laboral. Também, reduzir e prevenir riscos e, assim, proporcionar melhor condição de saúde. ${ }^{19,22}$

0 instrumento permite ter uma visão ampliada do trabalhador voltada para: riscos ocupacionais; estilo de vida; hábitos de manutenção ou não da saúde e a influência do ambiente de trabalho nos aspectos biológicos, espirituais, desenvolvimentais e psicossociais da vida dos trabalhadores. Ele deve estar em consonância com a Teoria de Enfermagem escolhida, bem como com a legislação que regulamenta a SAE e o PE. ${ }^{12,19,22}$

\section{CONSIDERAÇÕES FINAIS}

O presente estudo permitiu a construção de um instrumento contendo três impressos para subsidiar a consulta de enfermagem a trabalhadores por meio do processo de enfermagem. Foram identificados os possíveis diagnósticos, intervenções e resultados de enfermagem, portanto, o objetivo proposto foi alcançado.

Como contribuições deste estudo para a enfermagem e a saúde do trabalhador, têm-se: criação de um instrumento para subsidiar a consulta de enfermagem a trabalhadores; atendimento à legislação vigente relativa à implementação e registros do processo de enfermagem.

0 instrumento permite ainda um mapeamento dos grupos de risco e permite priorizar as intervenções e ações de promoção da saúde, a partir da Teoria de Betty Neuman. Como limitação deste estudo, tem-se que o instrumento proposto não foi 


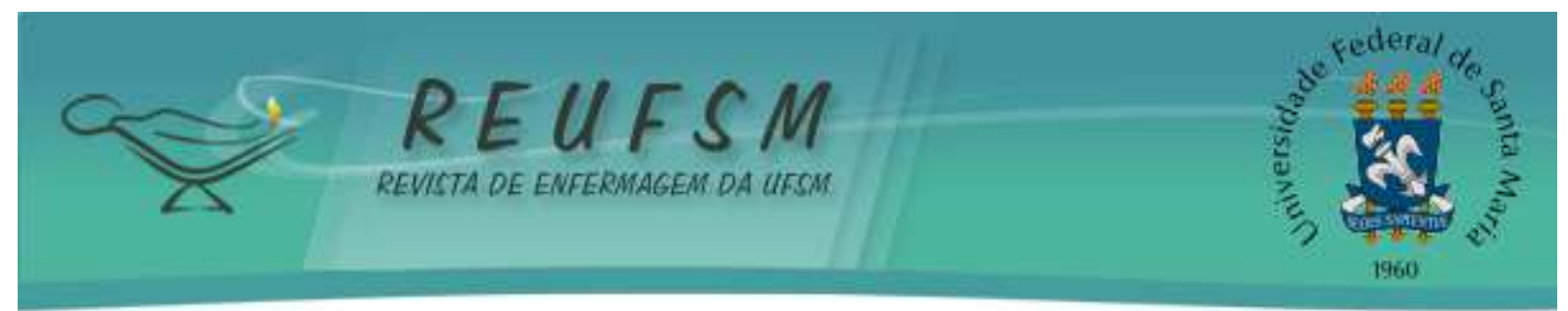

aplicado e validado na prática clínica. Assim, sugere-se sua validação de conteúdo e clínica com adequação para a realidade e especificidade de cada instituição.

\section{REFERÊNCIAS}

1. Governado do Estado de Minas Gerais. Secretaria Estadual de Saúde de Minas Gerais. Construindo ações de Saúde do Trabalhador no âmbito das Superintendências e Gerências Regionais de Saúde. Belo Horizonte; 2011.

2. Silva CO, Ramminger T. O trabalho como operador de saúde. Ciênc Saúde Coletiva [Internet]. 2014 [acesso em 2015 mar 10];19(12):4751-8. Disponível em: http: / / www.scielo.br/scielo.php?script=sci_arttext\&pid=S1413-81232014001204751 .

3. Pinto VLX, Moreira CVS, Bezerra IWL, Pequeno NPF. Labor, trabalho e ação: elementos pertinentes aos conceitos arendtianos em relatos autobiográficos de trabalhadores do setor de transportes. Saúde Soc [Internet]. 2014 [acesso em 2015 fev 20];23(4):1288-300. Disponível em: http: //www.scielo.br/pdf/sausoc/v23n4/0104-1290-sausoc-23-4-1288.pdf.

4. Silva CT, Terra MG, Camponogara S, Kruse MHL, Roso CC, Xavier MS. Educação permanente em saúde a partir de profissionais de uma residência multidisciplinar: estudo de caso. Rev Gaúch Enferm [Internet]. 2014 [acesso em 2015 fev 12];35(3):49-54. Disponível em: http://seer.ufrgs.br/index.php/RevistaGauchadeEnfermagem/article/view/44512/31504.

5. Minghetti LR, Kanan LA, Rocha G. Saúde mental do trabalhador: transtornos mentais e do comportamento relacionados com o trabalho que podem, em alguma medida, estar associados ao suicídio laboral. RIES. 2014;3(1):105-43.

6. Borges AM, Silva DF, Almeida MCV, Rocha LP, Bonow CA, Cezar-Vaz MR. Avaliação do ensino em saúde do trabalhador por acadêmicos de enfermagem. Rev Pesqui Cuid Fundam Online [Internet]. 2014 [acesso em 2015 mar 08];6(4):1349-60. Disponível em: http://www.seer.unirio.br/index.php/cuidadofundamental/article/view/3324/pdf_649.

7. Nunes ECDA, Silva LWS, Pires EPOR. O ensino superior de enfermagem: implicações da formação profissional para o cuidado transpessoal. Rev Latinoam enferm [Internet]. 2011 [acesso em 2015 jan 18];19(2):252-60. Disponível em: http://www.scielo.br/pdf/rlae/v19n2/pt_05.pdf.

8. Almeida MCV, Silva MRS, Borges AM, Rocha LP, Cezar-Vaz MR. Elementos ecossistêmicos da saúde do trabalhador portuário e seu processo de trabalho. Ciênc Cuid Saúde [Internet]. 2014 [acesso em 2015 fev 23];13(4):764-9. Disponível em: http://periodicos.uem.br/ojs/index.php/CiencCuidSaude/article/view/19109/pdf_253.

9. Ribeiro MCS. Operacionalização das ações de saúde do trabalhador no quotidiano da enfermagem. In: Ribeiro MCS, organizador. Enfermagem e trabalho: fundamentos para a atenção à saúde dos trabalhadores. São Paulo: Martinari; 2008.

10. Sotero ACS, Lungwtz MP, Pauferro NBC, Lanza LB. Aplicação de um instrumento de registro da assistência sistematizada de enfermagem em saúde ocupacional em uma empresa de metalurgia. Rev Fac Cienc Med Sorocaba [Internet]. 2013 [acesso em 2015 mar 27];15(4):109-11. Disponível http://revistas.pucsp.br/index.php/RFCMS/article/view/12884/pdf.

11. Ferreira GC, Arreguy-Sena C, Alves MS, Salimena AMO. Captação de órgãos e o binômio familiares/corpo: instrumentos para subsidiar a abordagem do enfermeiro. Rev RENE 


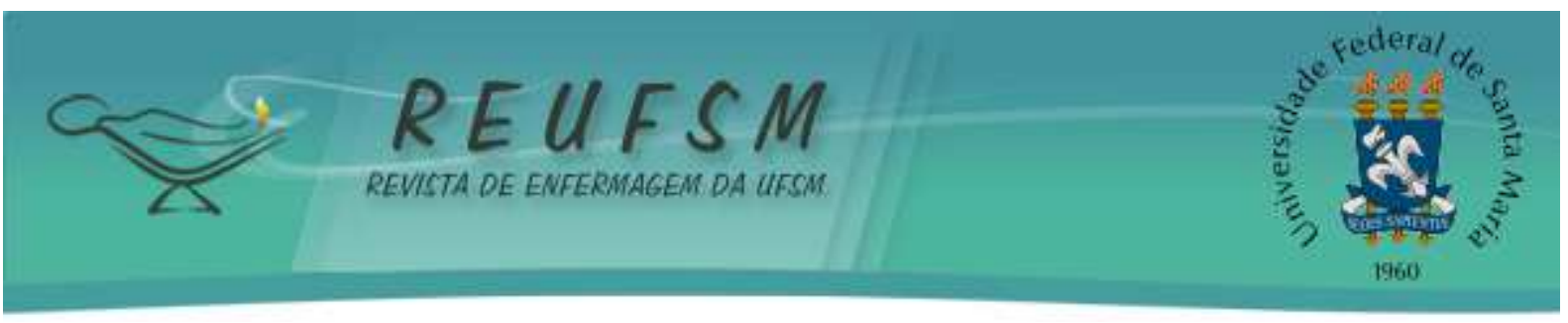

[Internet]. 2013 [acesso em 2014 jul 20];14(2):405-16. Disponível em: http://www.revistarene.ufc.br/revista/index.php/revista/article/view/1295.

12. Conselho Federal de Enfermagem (COFEN). Resolução COFEN $n^{\circ} 358$, de 15 de outubro de 2009. Dispõe sobre a Assistência de Enfermagem e a implementação do Processo de Enfermagem em ambientes, públicos ou privados, em que ocorre o cuidado profissional de enfermagem, e dá outras providências. Brasília (DF): COFEn; 2009.

13. Alvarenga-Martins N, Arreguy-Sena C, Alves MS, Moura DCA. Teoria de intersistemas de Bárbara Artinian na consulta de enfermagem junto à pessoa com lesão de pele. CuidArte Enferm [Internet]. 2015 [acesso em 2016 ago 21];9(1):9-17. Disponível em: http://fundacaopadrealbino.org.br/facfipa/ner/pdf/Revistacuidarteenfermagem\%20v.\%20 9\%20n.1\%20\%20jan.\%20jun\%202015.pdf.

14. NANDA Internacional/Diagnósticos de enfermagem da NANDA: definições e Classificação 2009-2011. Porto Alegre (RS): Artmed; 2010.

15. Bulechek GM, Butcher HK, Dochterman JM. Classificação das Intervenções de Enfermagem (NIC). 5a ed. Rio de Janeiro (RJ): Elsevier; 2010.

16. Moorhead S, Johnson M, Maas ML, Swanson E. Classificação dos Resultados de Enfermagem (NOC). $4^{\mathrm{a}}$ ed. Rio de Janeiro (RJ): Elsevier; 2010.

17. Arreguy-Sena C, Carvalho ECd, Rossi LA, Rufino MC. Estratégias de implementação do processo de enfermagem para uma pessoa infectada pelo HIV. Rev Latinoam Enferm. 2001;9(1):27-38.

18. Alvarenga-Martins N, Romera DS, Silva DVB, Alampi FF, Gomes JJ, Silva D. Teoria de Betty Neuman na abordagem de pessoas com Grangrena de Fournier. Arq Cienc Saúde [Internet]. 2016 [acesso em 2016 ago 21];23(2):92-9. Disponível em: http://www.cienciasdasaude.famerp.br/index.php/racs/article/view/699/pdf_111.

19. McEwen M, Wills EM. Bases teóricas para enfermagem. $2^{\mathrm{a}}$ ed. Porto Alegre: Artmed; 2009.

20. Greco RM, Moura DCA, Sena CA, Martins NA, Alves MS. Labour conditions and theory of Betty Neuman: third-party workers of a public university. J Nurs UFPE on line [Internet]. 2016 [acesso em 2016 ago 21];10(Suppl. 2):727-35. Disponível em: http://www.revista.ufpe.br/revistaenfermagem/index.php/revista/article/view/7706/pdf -9682.

21. Alfaro-Lefreve R. Aplicação do processo de enfermagem: um guia passo a passo. $5^{\mathrm{a}}$ ed. Porto Alegre: Artes Médicas; 2005.

22. George JB. Teorias de Enfermagem: fundamentos a prática profissional. $4^{a}$ edição. Porto Alegre: Artmed; 2000.

23. Carvalho GM. Enfermagem do trabalho. $2^{\mathrm{a}}$ ed. Rio de Janeiro: Guanabara Koogan; 2014.

Data de recebimento: 06/10/2015

Data de aceite: 13/12/2016

Contato do autor responsável: Denise Cristina Alves de Moura. Endereço postal: Rua Marechal Floriano Peixoto, $n^{\circ}$ : 509, apartamento: 403, Centro. CEP: 36015-440. Juiz de Fora, Minas Gerais, Brasil. E-mail: denisematipo@yahoo.com.br 